This is an electronic reprint of the original article. This reprint may differ from the original in pagination and typographic detail.

Author(s): Koivistoinen, Juha; Sládková, Lucia; Aumanen, Jukka; Koskinen, Pekka; Roberts, Kevin; Johansson, Andreas; Myllyperkiö, Pasi; Pettersson, Mika

Title: $\quad$ From Seeds to Islands: Growth of Oxidized Graphene by Two-Photon Oxidation

Year: $\quad 2016$

Version:

Please cite the original version:

Koivistoinen, J., Sládková, L., Aumanen, J., Koskinen, P., Roberts, K., Johansson, A., Myllyperkiö, P., \& Pettersson, M. (2016). From Seeds to Islands: Growth of Oxidized Graphene by Two-Photon Oxidation. Journal of Physical Chemistry C, 120(39), 2233022341. https://doi.org/10.1021/acs.jpcc.6b06099

All material supplied via JYX is protected by copyright and other intellectual property rights, and duplication or sale of all or part of any of the repository collections is not permitted, except that material may be duplicated by you for your research use or educational purposes in electronic or print form. You must obtain permission for any other use. Electronic or print copies may not be offered, whether for sale or otherwise to anyone who is not an authorised user. 


\title{
From Seeds to Islands: Growth of Oxidized \\ Graphene by Two-Photon Oxidation
}

\author{
Juha Koivistoinen, ${ }^{1}$ Lucia Sladkova, ${ }^{1,2}$ Jukka Aumanen, ${ }^{1}$ Pekka Koskinen, ${ }^{3}$ Kevin Roberts, ${ }^{3}$ \\ Andreas Johansson, ${ }^{1,3}$ Pasi Myllyperkiö, ${ }^{1}$ Mika Pettersson ${ }^{1 *}$ \\ ${ }^{1}$ Nanoscience Center, Department of Chemistry, P.O. Box 35, FI-40014, University of Jyväskylä, \\ Finland \\ ${ }^{2}$ CEITEC - Central European Institute of Technology, Purkynova 123, Brno 61200, Brno \\ University of Technology, Czech Republic
}

${ }^{3}$ Nanoscience Center, Department of Physics, P.O. Box 35, FI-40014, University of Jyväskylä, Finland

ABSTRACT. Mechanism of two-photon induced oxidation of single-layer graphene on $\mathrm{Si} / \mathrm{SiO}_{2}$ substrates is studied by atomic force microscopy (AFM) and Raman microspectroscopy and imaging. AFM imaging of areas oxidized by using a tightly focused femtosecond laser beam shows that oxidation is not homogeneous but oxidized and non-oxidized graphene segregate into separate domains over the whole irradiated area. Oxidation process starts from point-like "seeds" 
which grow into islands finally coalescing together. The size of islands before coalescence is 30 $40 \mathrm{~nm}$ and the density of the islands is on the order of $10^{11} \mathrm{~cm}^{-2}$. Raman spectroscopy reveals growth of the $\mathrm{D} / \mathrm{G}$ band ratio along the oxidation. Sharpness of the D-band which persists over large range of oxidation and the maximal value of the intensity ratio of the D- and G-bands ( 0.8) indicates that graphene oxidation proceeds by increase of the oxidized area rather than progression of oxidized areas to fully disordered structure. A phenomenological model is developed which explains the observations. According to the model, the probability for oxidation of a site next to already oxidized site is five orders of magnitude higher than oxidation of pristine graphene. Irradiation of an extended area by raster scanning leads to a formation of an irregular nanomesh of oxide islands with a narrow size distribution. The phenomenological model yields similar results as the experiment. This study forms a basis for controlled use of two-photon oxidation for tailoring properties of graphene and patterning it with sub-micrometer resolution.

INTRODUCTION. A promising application area of graphene is transparent and flexible electronics. ${ }^{1-3}$ Being practically transparent but still possessing extremely high charge carrier mobility combined with potential for tuning of electronic properties forms an attractive platform for development of novel applications. It has been routinely showed that graphene can be used to fabricate field-effect transistors when combined with conventional lithographic fabrication methods and metal-based structures and top- or bottom gates. ${ }^{1-3}$ However, an alternative scenario is all-graphene technology which takes advantage of potential tunability of electronic properties of graphene. This development is at its infancy and much work is needed before such approach will be realized into useful applications. The key question is how to modify the properties of 
graphene with sufficient spatial resolution cost-efficiently and scalably. To this end, laser based direct writing offers interesting possibilities. By using laser reduction of graphene oxide (GO), functional devices including transistor, supercapacitor and photodetector have been demonstrated. ${ }^{4-8}$ However, significant restriction in this approach is that reduction of GO does not recover excellent electronic properties of graphene, which degrades performance of devices compared to the graphene based approach. Recently, we presented an opposite approach by performing laser-induced oxidation of graphene with a two-photon mechanism using short femtosecond pulses. ${ }^{9,10}$ Opening of a band gap in graphene was demonstrated as a result of oxidation, which could be controlled by adjusting irradiation parameters. This observation is in agreement with several studies which show that the band gap of GO depends on the level of oxidation. ${ }^{11,12}$ Thus, the presented method forms an excellent basis for light-induced fabrication of all-graphene devices. However, for accurate control of the process, basic knowledge on the oxidation process is needed. Structure and composition of GO is ill-defined and depends on the method of preparation. ${ }^{13}$ In the nanoscale, it has been found that GO is heterogeneous material consisting of graphene-like and oxide-like regions. However, it is not clear if the GO produced by the two-photon oxidation is similar to GO produced by chemical methods. In order to exploit the direct laser writing method further, more information on the process is needed.

In this study, we investigate GO produced by two-photon oxidation. Systematic variation of irradiation parameters combined with AFM and Raman measurements gives a detailed picture of the formation process. We develop a phenomenological model which successfully reproduces the observations. The results of this study can be used for controlling two-photon oxidation of graphene in order to yield material with tailored properties. This, in turn, can be used for developing all-graphene devices for various applications. 


\section{EXPERIMENTAL SECTION}

Sample Fabrication. A silicon chip $\left(10 \mathrm{~mm}\right.$ x10 mm) with a top layer of $\mathrm{SiO}_{2}$ (300 nm thick) and a monolayer of CVD synthesized graphene was purchased from Graphenea. On top of the chip a grid pattern was made to serve as a reference to locate oxidation patterns during the study. Using PMMA and electron beam lithography, a grid pattern with 10 x 10 squares of size $200 \mu \mathrm{m}$ x $200 \mu \mathrm{m}$ each was fabricated. The line width of the grid pattern was $1 \mu \mathrm{m}$. The chip was treated with light oxygen plasma (Oxford Plasmalab 80 reactive ion etcher) to remove residual PMMA and graphene at the bottom of the grid pattern before metallization through physical vapor deposition with $2 \mathrm{~nm}$ of $\mathrm{Ti}$ and $30 \mathrm{~nm}$ of Au. The silicon chip was again covered with a protective layer of PMMA and diced to $5 \mathrm{~mm}$ x $5 \mathrm{~mm}$ pieces, using a Loadpoint MicroAce 3 dicing saw. The chip was then cleaned in several steps. First the top PMMA layer with silicon dust was rinsed off with acetone. Then lift-off was performed in hot acetone to remove the metallization on top of PMMA. Finally the chip was again spin coated with PMMA and kept in acetone for an hour, then carefully rinsed in isopropanol and dried with a dry nitrogen gun. Inspection in optical microscope showed no obvious PMMA residues on the surface.

Photo-oxidation and FWM imaging. The laser setup for four-wave mixing imaging and twophoton oxidation is described in detail in our previous article. ${ }^{9}$ Briefly, an amplified femtosecond laser (Pharos-10, $600 \mathrm{kHz}$, Light Conversion) was used to pump two non-collinear optical parametric amplifiers (NOPAs, Orpheus-N, Light Conversion). The two beams were combined to collinear geometry and focused on the sample by a microscope objective (Nikon LU Plan ELWD 100x/0.80). The group velocity dispersion of the pulses was compensated with a prism compensator. Photo-oxidized spots were produced on monolayer graphene on doped silicon 
substrate with dielectric silicon dioxide layer (Graphenea). The oxidized spots were produced by tight focusing (diameter $\sim 500 \mathrm{~nm}$ ) of a laser beam on the graphene sample. Oxidation was performed under ambient air with $540 \mathrm{~nm}$ laser pulses of $40 \mathrm{fs}$ pulse duration and $600 \mathrm{kHz}$ repetition rate. 16 different pulse energy and irradiation time combinations were used for oxidation. With each parameter set 25 spots in $5 x 5$ matrix with $1 \mu \mathrm{m}$ spot separation were oxidized.

The oxidized area was imaged by using FWM imaging which was performed under nitrogen purge using 540 and $590 \mathrm{~nm}$ laser beams and 3 pJ pulse energy. The generated $500 \mathrm{~nm}$ FWM signal was collected to backscattering direction and spectrally separated from the excitation beams by using optical filters. The signal was collected by a photon counting module (single photon avalanche photodiode, SPCM-AQRH-14, Excelitas Technologies).

AFM. The AFM measurements were performed with Dimension 3100 (Digital Instruments, Veeco) in tapping mode. The measured area was in the range $5 \times 5 \mu^{2}$ (1024x1024 pixels) to cover the area oxidized with the same pulse energy and irradiation time combination. The smaller area $3.5 \times 3.5 \mu \mathrm{m}^{2}$ (896x896 pixels) was then chosen for more precise analysis of the oxidized spots. The scanning frequency was adjusted according to the required picture resolution and tip conditions. The lateral AFM resolution is limited by the convolution of the tip shape (with a nominal radius of $8 \mathrm{~nm}$ ) and the shape of the oxidized islands. The lateral resolution is here estimated by the FWHM from the cross-section of the smallest islands imaged, giving a value close to $8 \mathrm{~nm}$.

Raman spectroscopy and imaging. Raman measurements were performed with home-built Raman setup in a backscattering geometry using $532 \mathrm{~nm}$ excitation wavelength produced with CW single frequency laser (Alphalas, Monolas-532-100-SM). The beam was focused to a sample 
and the signal was collected with a 100x microscope objective (Nikon L PLAN 100x with 0.70 N.A.). The scattered light was dispersed in a $0.5 \mathrm{~m}$ imaging spectrograph (Acton, SpectraPro 2500i) using $600 \mathrm{~g} / \mathrm{mm}$ grating (resolution: $7-8 \mathrm{~cm}^{-1}$ ). The signal was detected with EMCCD camera (Andor Newton EM DU971N-BV) using $100 \mu \mathrm{m}$ slit width. A beam splitter was placed between the objective and the spectrometer in order to observe the exact measurement point visually. The Rayleigh scattering was attenuated with an edge filter (Semrock). The sample positioning was done with XYZ-piezoscanner (ThorLabs, NanoMax 300) with a smallest step of $5 \mathrm{~nm}$ in each direction. Laser power of $1.5 \mathrm{~mW}$ was utilized and the mapping was conducted using $200 \mathrm{~nm}$ step size and 3 s accumulation time in $20 \mu \mathrm{m}$ x $20 \mu \mathrm{m}$ area.

Graphene deposited on $\mathrm{Si} / \mathrm{SiO}_{2}$ substrate was confirmed by Raman measurements as single layer graphene with partial local inhomogeneities resulting from ripples, defects and grain-like partial double layer structure.

\section{RESULTS}

Four wave mixing imaging. FWM image of the oxidized area is presented in Fig. 1 (A) and a close-up of the region in the upper right corner is shown in (B). Pristine monolayer graphene has purple color in the image, whereas the bright orange/yellow spots are the areas with double or multilayer graphene. The areas with different oxidation parameters are illustrated with a grid with yellow dashed lines. The map of oxidized spots and the used irradiation parameters is shown in Fig 1(C). The oxidation time increases from left to right (0.2, 0.5, 1.0 and $2.0 \mathrm{~s} / \mathrm{spot})$ and pulse energy (8.3, 10.5, 13.2 and $16.6 \mathrm{pJ} /$ pulse) increases from the bottom to top. The most strongly oxidized spots that are located on the upper right corner appear as black spots in the 
FWM image, but most of the oxidations are not clearly visible. The middle point of the rightmost column of the longest irradiations was intentionally irradiated 22 seconds instead of two seconds. These stronger oxidations helped us to locate the patterns with AFM and FWM imaging. These spots can be seen on the right edge of the FWM image.

(B)

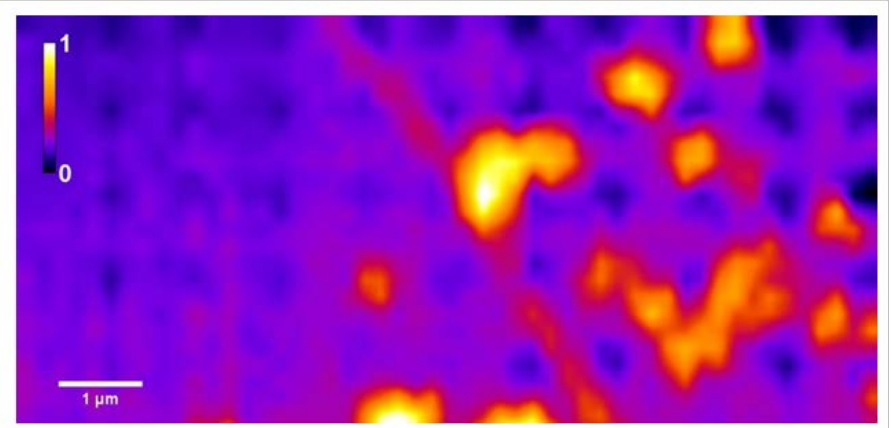

(A)

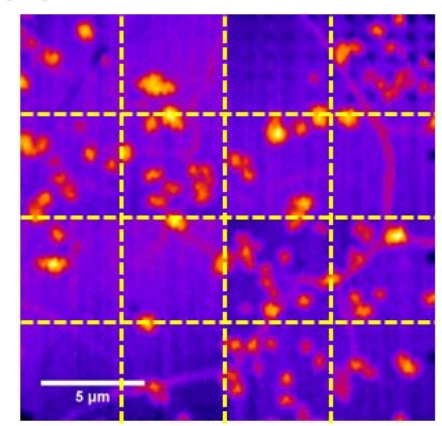

(C)

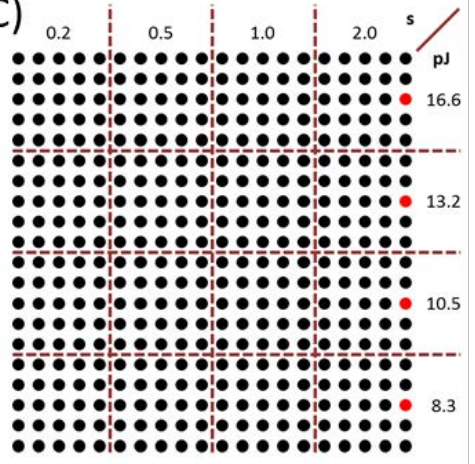

Figure 1. Four wave mixing (FWM) image of the graphene sample (A), a close-up of the upper right area (B) and a map showing the pattern of the oxidized spots (C). Oxidation time and pulse energy for the spots are shown in each column and row, respectively. The red spots were oxidized 22 s/spot. The irradiated spots are visible in the FWM image as dark spots on graphene and they are most clearly visible on the upper right corner of the image which was irradiated with the highest dose. The darkness level correlates with irradiation dose which varies quadratically with laser intensity (due to two-photon process) and linearly with irradiation time. 
The bright spots and lines are due to double layer graphene, folds, and grain boundaries or other imperfections.

AFM imaging. An AFM image of the oxidized sample is shown in Fig. 2(A) It shows the 25 spots that were irradiated with $13.2 \mathrm{pJ}$ pulse energy and the irradiation time of $2 \mathrm{~s} / \mathrm{spot}$. These spots can be clearly distinguished from the background roughness which is due to residues left from the transfer process of graphene. The corresponding FWM image is in the second row from above of the rightmost column in Fig. 1. The irradiated spots are clearly visible as round textured areas with increased height compared to graphene base level (See also Fig. 2(B)). The diameter of the areas is about 300 - $500 \mathrm{~nm}$ which corresponds well with the diffraction limited spot of $500 \mathrm{~nm}$ combined with a two-photon oxidation process which makes the spot effectively smaller than the real laser spot size. The height of the irradiated material is $1-2 \mathrm{~nm}$ larger than graphene as shown in Fig. 2(C). A fit to the cross-section yields a diameter of $400 \mathrm{~nm}$ for the oxidized spot. The textured areas are surrounded by “clean” flat areas which are distinguishable from the rough features which are due to polymer residues remaining from the graphene transfer process. It is notable that the texture of the irradiated spots is markedly different from the polymer residues. The third highest spot on the right was irradiated much longer than the other spots (22 s) with the result that the texture has transformed into a smooth area. In the middle of the oxidized spot degradation of the oxidized structure occurs - the middle part was lower than the outer part. 

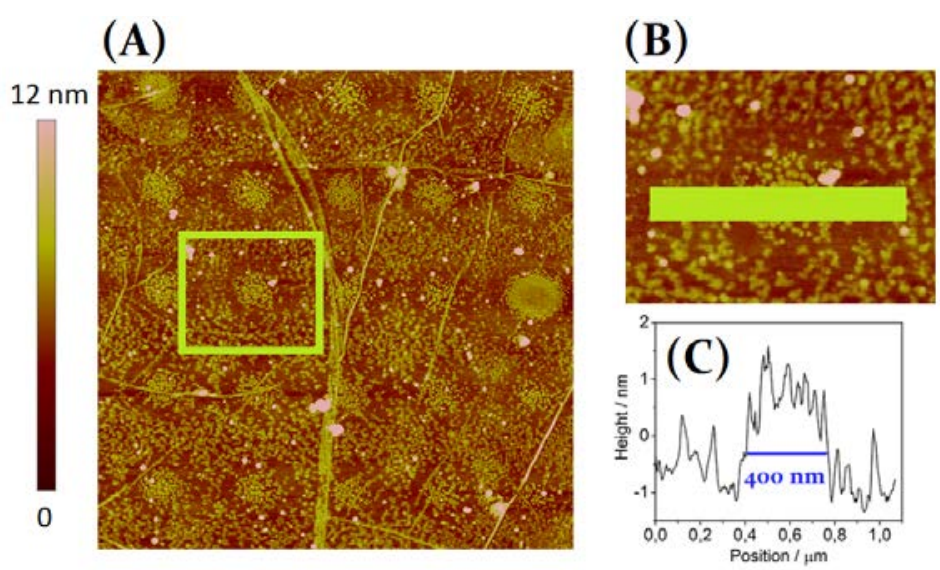

Figure 2. (A) AFM image of graphene with irradiated spots, (B) a close-up of the area marked in (A) with a rectangle, (C) a cross-section of the line shown in (B). The irradiation pulse energy was $13.2 \mathrm{pJ}$ and the irradiation time was $2 \mathrm{~s}$, except for the third highest spot on the right which was irradiated for $22 \mathrm{~s}$.

Closer inspection of the irradiated spots with AFM shows that oxidation is not homogeneous inside the irradiated areas but there are small islands inside the irradiation spot. The time evolution of the islands was studied by imaging spots after various doses of irradiation. AFM images from several spots irradiated with various doses of $8.3 \mathrm{pJ}$ pulses are shown in Figures 3 (A)-(D). At least four different spots at each irradiation dose were prepared and measured and the repeatability of the results was confirmed. The images in Fig. 3 represent well the general behavior. The spot with the lowest irradiation dose (0.2 s, Fig. (3(A)) shows that the irradiation area has been "cleaned" from residues by irradiation and a number of small islands have emerged with apparently random arrangement. Although the polymer residues are also present on the sample, the irradiation-induced islands are easily distinguished from them due to cleaned area and different morphology of the islands. By inspection of images 3(A) - (D) it is observed that with prolonged irradiation, more islands appear and they grow. The growth is fastest in the 
center part of the irradiated spot due to the higher intensity of the Gaussian beam at the beam center and the trend is further amplified by the two-photon nature of the oxidation process. ${ }^{9}$ This is particularly evident in the Fig. 3(D) where the islands in the center area already coalesce together while on the edges they are still rather small and well separated. When islands have grown to a size of approximately $30 \mathrm{~nm}$ in diameter, they start coalescing together. It should be noted that the size estimate is probably somewhat too low due to the finite lateral resolution of AFM (8 nm). Thus, a safer estimate is thus $30-40 \mathrm{~nm}$. This behavior is qualitatively similar for different irradiation pulse energies except that time evolution occurs faster for higher irradiation intensities.
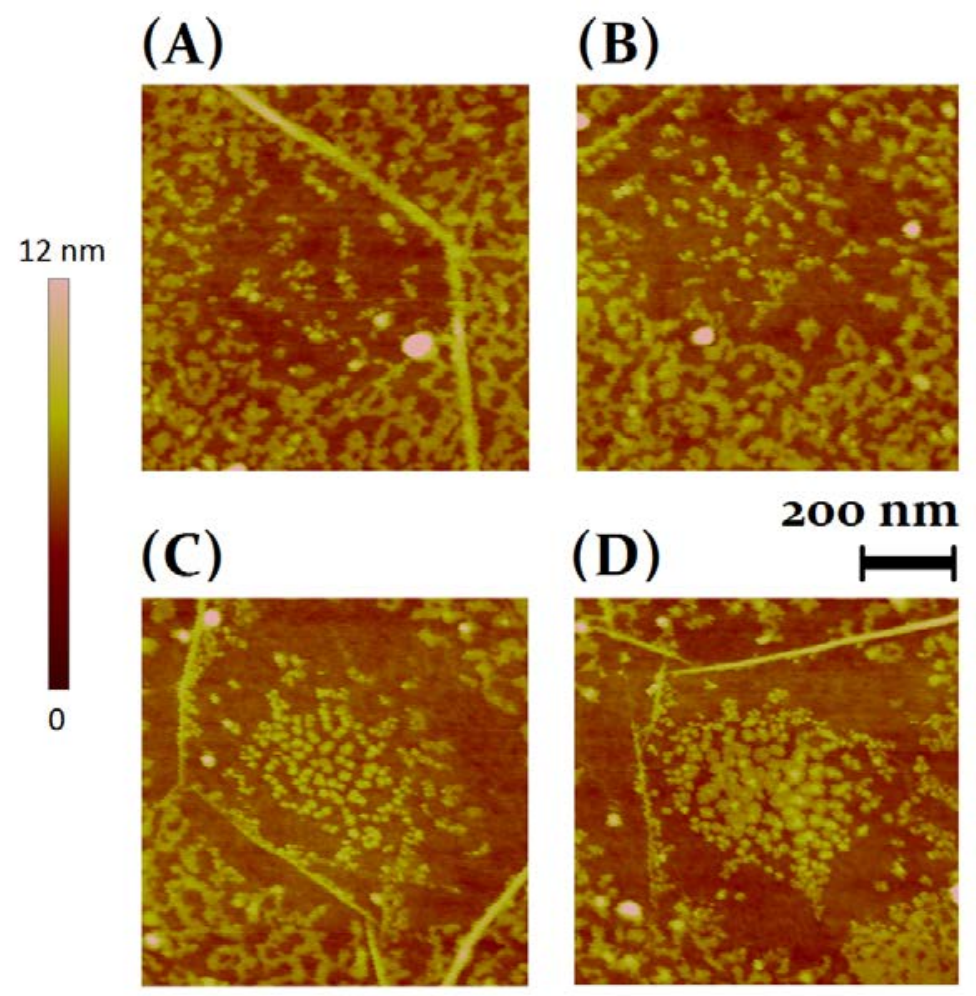

Figure 3. AFM images of irradiates spots on graphene. The pulse energy was $8.3 \mathrm{pJ}$ and the irradiation time was $0.2 \mathrm{~s}(\mathrm{~A}), 0.5 \mathrm{~s}(\mathrm{~B}), 1 \mathrm{~s}(\mathrm{C})$ and $2 \mathrm{~s}(\mathrm{D})$. 
The number density of islands is limited by the coalescence after they grow large enough. Due to this, after the initial increase of the number density, it saturates to a value which is fairly similar in all the cases studied here, except that for the highest pulse energy (16.6 pJ) the number was somewhat larger (up to a factor of two). The average saturated number density from several measurements was 87 islands per spot. The spot diameter enclosing the islands was about 400 $\mathrm{nm}$ for high doses, which yields an average number density of $6.9 \times 10^{10} \mathrm{~cm}^{-2}$. The accuracy of this estimate is limited by the fact that as soon as two islands touch each other they were calculated as one island. If one would try to estimate the total number of initially created islands, the number would be probably somewhat larger.

Statistics of the island growth dynamics was studied by converting the AFM images to binary figures and performing image analysis. Distribution of islands with varying size at $0.2 \mathrm{~s}$ and $2 \mathrm{~s}$ irradiation times at 8.3 pJ pulse energy are presented in Figures 4(A) and 4(B), respectively. The data is a sum from four different spots at each irradiation time. The data shows that in both cases the distribution peaks at smallest island size but, at the same time, the proportion of larger islands grows at higher irradiation times. The corresponding data at different irradiation intensities are qualitatively similar as seen in Fig 4(C) which shows normalized distributions in the same plot.

Important information considering the island growth mechanism is provided by the growth kinetics. This was analyzed by plotting the total area of islands as a function of irradiation time for different irradiation intensities. The result is shown in Fig. 5(A). When comparing the kinetic data for different pulse energies one has to take into account that the photo-oxidation process occurs via two-photon mechanism. Accordingly, the irradiation dose is proportional to the square of the pulse energy. Therefore, we plot the data of Fig 5(A) in Fig. 5(B) arranged so that the x- 
axis is $E^{2} t$, where $E$ is pulse energy and $t$ is irradiation time. From now on, we call this quantity as "irradiation dose".
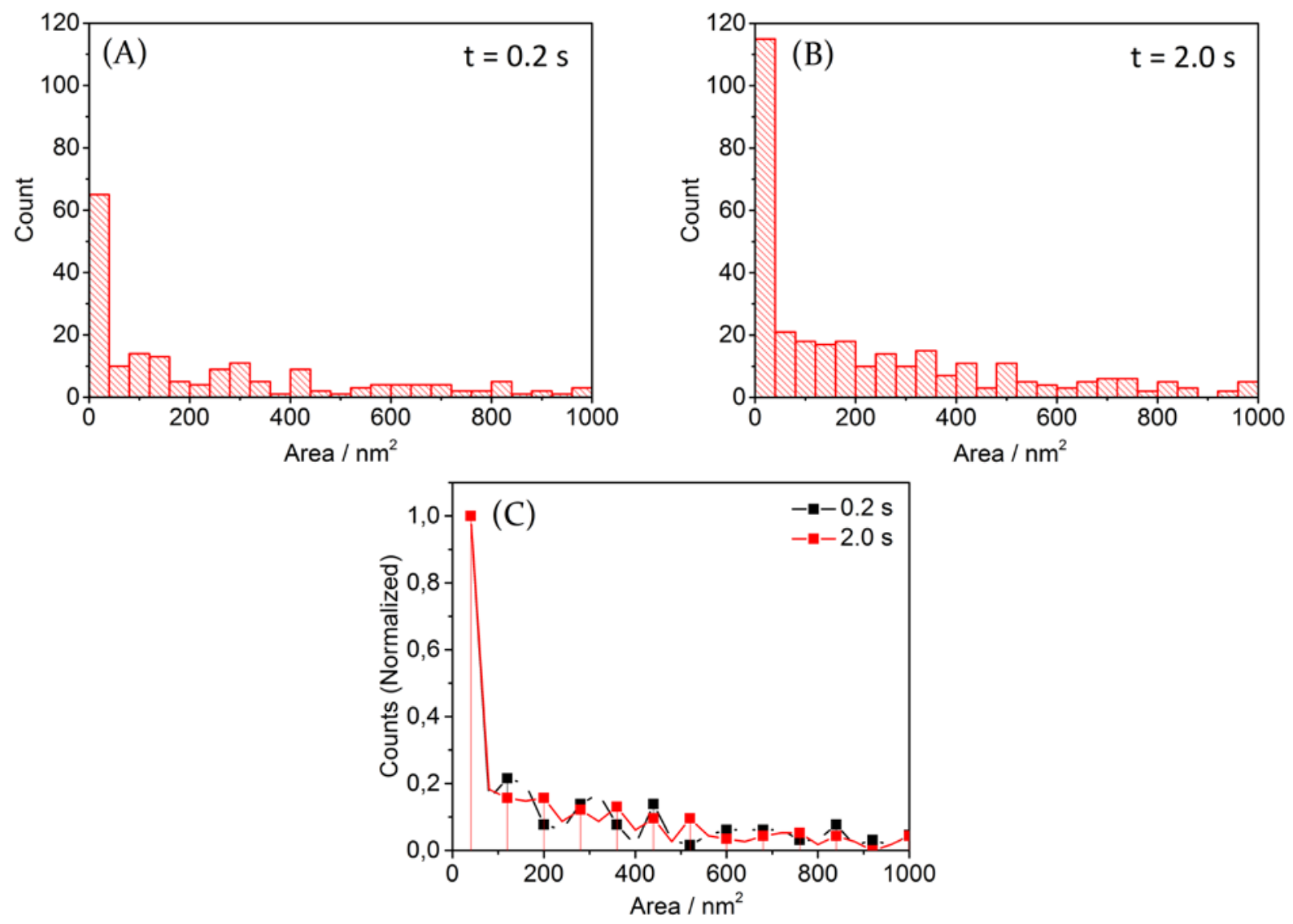

Figure 4. Histograms of the number of islands as a function of island area for $8.3 \mathrm{pJ}$ pulse energy and $0.2 \mathrm{~s} \mathrm{(A)} \mathrm{and} 2 \mathrm{~s}(\mathrm{~B})$ irradiation time. Both data are showed normalized in (C). Note that assuming round islands, the areas of 20 and $30 \mathrm{~nm}$ diameter islands correspond to 314 $\mathrm{nm}^{2}$ and $707 \mathrm{~nm}^{2}$, respectively. 

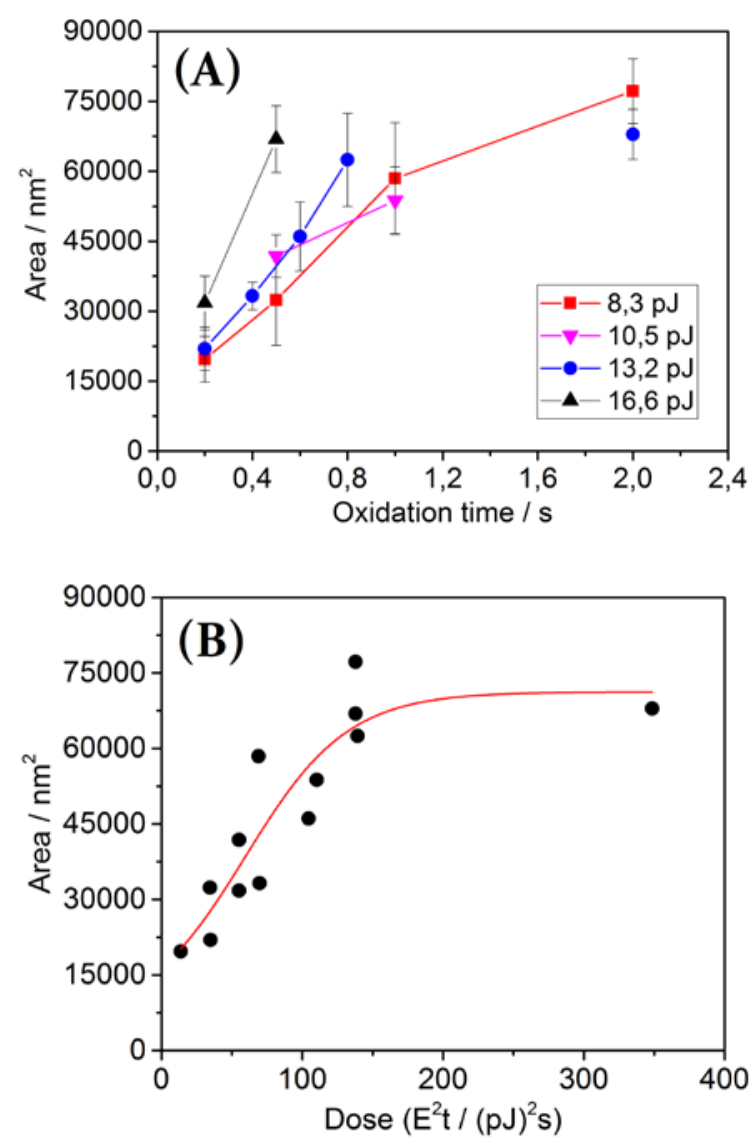

Figure 5. Growth kinetics of oxidized islands. (A) Total area of the islands as a function of irradiation time at different pulse energies. (B) The same data as in Fig. 5A but the x-axis is represented as $E^{2} t(E$ = pulse energy, $t$ = irradiation time) in order to take into account the twophoton mechanism of photo-oxidation. This allows for combining data from different pulse energies. The red curve is fitted to the experimental data and has the functional form given by the phenomenological model described later (Eqs. (9) and (10)).

Raman imaging and spectroscopy. Raman imaging and spectroscopy was performed for a sample prepared similarly to the one which was used in AFM analysis. Raman image of the sample is shown in Figure 6. The intensity represents the D/G-band ratio showing that the irradiation increases the ratio as a function of irradiation dose. This is expected since it is known 
that functionalization, and oxidation in particular, leads to the growth of the D-band. ${ }^{13-15}$ The spots which have been irradiated less than $0.5 \mathrm{~s}$ are not well visible in the image showing that for the lowest irradiation doses the D-band growth is not detectable in the Raman image. The heavily irradiated area shows that the base signal level between the spots is also increased. This does not mean that the area between the spots is oxidized but it is due to the Raman probe spot being too large to resolve spatially separate spots well enough. The apparent resolution is better in the FWM image (Fig. 1) since the nonlinearity of the method yields smaller effective spot size.

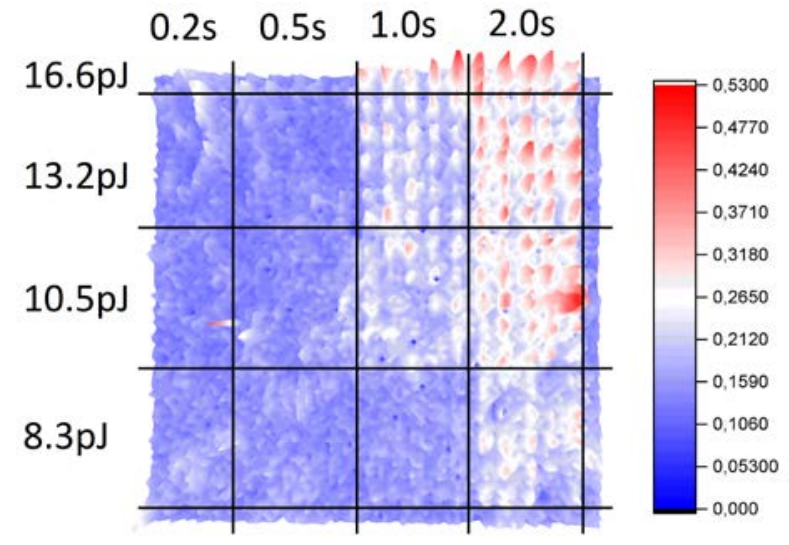

Figure 6. Raman map of oxidized spot matrix. The ratio of the intensities of the D- and G-bands is plotted.

Raman spectra were measured for various irradiation doses. In order to improve the signal to noise ratio, the spectra from the spot centers were averaged from each area having the same irradiation parameters. The Raman bands of the G-band, 2D-band and the D-band are shown in Figures 7(A), (B) and (C), respectively. 

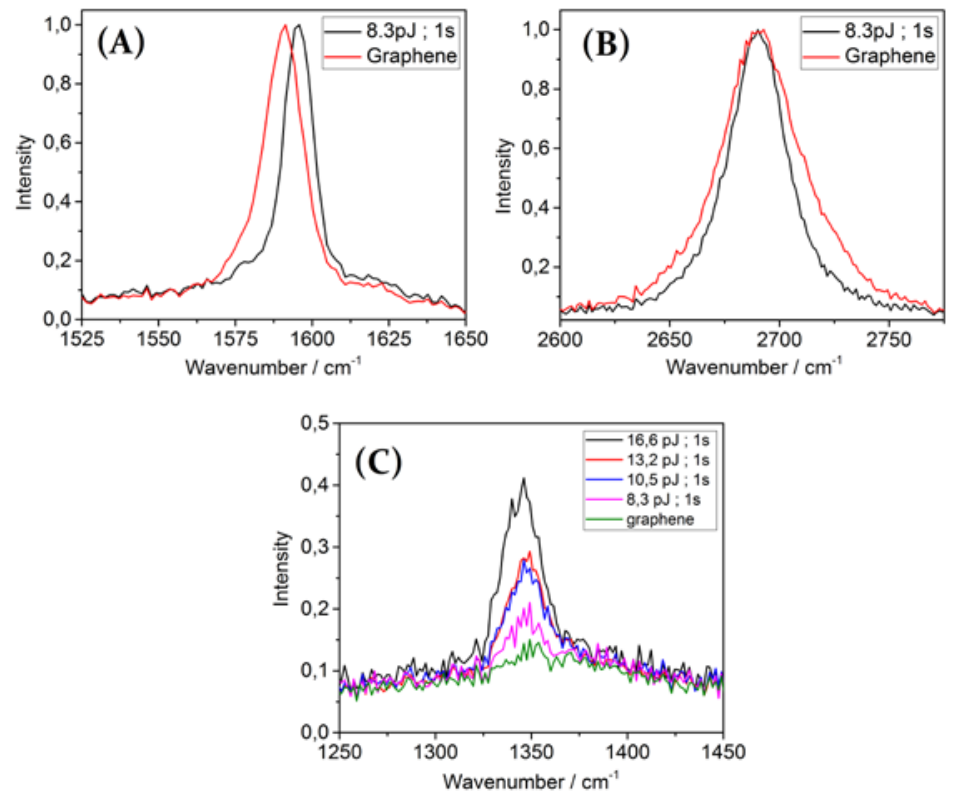

Figure 7. Raman bands of G- (A), 2D- (B) and D-bands of graphene and oxidized graphene. In (C) the intensities of the D-band are normalized according to the G-band.

It is clear from Fig. 7(A) that the G-band position shifts up and its width decreases upon irradiation. The 2D-band narrows as is evident in Fig 7 (B). The D-band grows slowly upon irradiation (Fig. 7 (C)). In line with this, the increase of the $\mathrm{D}^{\prime}$-band at $1620-1625 \mathrm{~cm}^{-1}$ is minute and hardly visible. A more detailed analysis of the Raman spectra was conducted by fitting single Lorentzian function to the 2D-band data and one or two Lorentzian functions to the $\mathrm{G}$ band data. The position and width of the G- and 2D-bands were also determined and the results are shown in Figures 8(A) and 8(B), respectively. The G-band position upshifts rapidly for lowest doses of irradiation and then remains rather constant finally showing a slight downshift at highest doses. The G-band and the 2D-band FWHM are rapidly reduced at low irradiation doses after which the FWHM of the G-band returns to the initial value slowly. The 
changes occur much more rapidly than the growth of the D-band which occurs over longer irradiation.

(A)

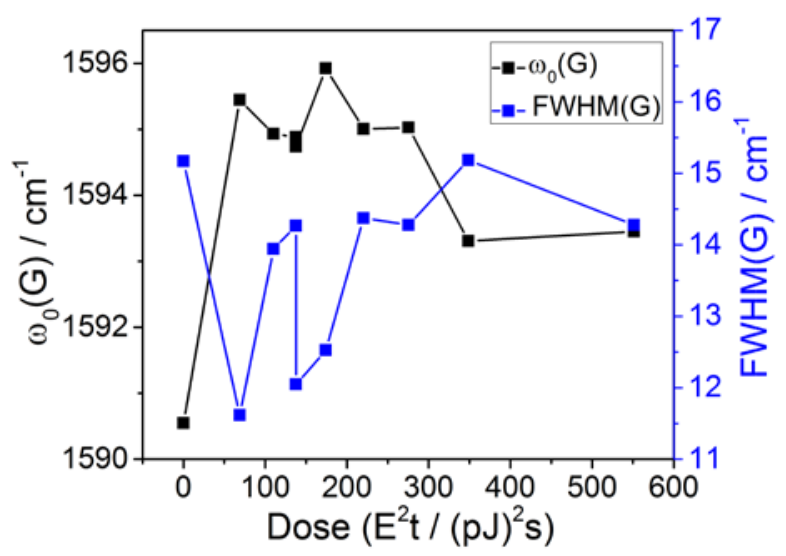

(B)

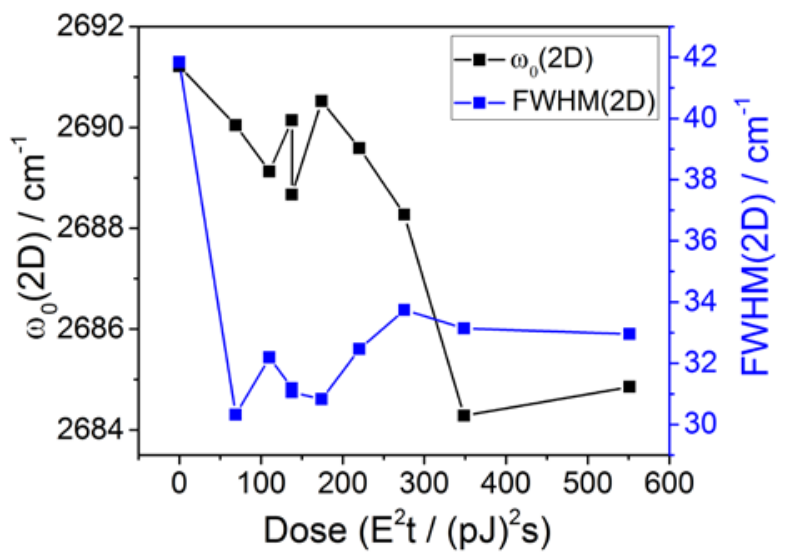

Figure 8. Raman data from oxidized spots: (A) G and (B) 2D-band Raman shift and FWHM with respect to dose.

Returning back to the apparently modest growth of the D-band (Fig. 7(C)), it seems to indicate that oxidation is not very extensive. The spectra show increased intensity of the D-band but the peak shape remains narrow throughout the whole irradiation range. Thus, there is no evidence of formation of highly disordered graphene which happens in particle irradiation when the defect density increases beyond the level of $10^{13} \mathrm{~cm}^{-2} .{ }^{16}$ Since oxidized graphene is segregated into islands we cannot use the $\mathrm{I}(\mathrm{D}) / \mathrm{I}(\mathrm{G})$ ratio directly to determine the defect density (corresponding to the degree of functionalization) within the oxidized areas. It would be possible to determine the average defect density if the fraction of the oxidized area compared to the total probed area was known. In this process, two factors should be bear in mind: the Raman beam is larger than the oxidized area, thus it probes areas outside the oxidation region. Secondly, as shown by AFM measurements, oxidized graphene grows as small islands and there is plenty of non-oxidized 
graphene in between them at early stages of oxidation. Thus, the Raman spectra are superpositions of the spectra from oxidized islands and from non-oxidized graphene. In order to extract the Raman spectra of oxidized areas a careful analysis was done. The analysis is based on the assumption that the total spectrum is a sum of pristine graphene $(G)$ and oxidized graphene (GO) weighted by their fractional areas:

$I^{O b s}(\bar{v})=c_{1} I^{G}(\bar{v})+c_{2} I^{G O}(\bar{v})$

Where Obs, G and GO refer to the measured spectrum, graphene and graphene oxide, respectively. The $c_{1}$ and $c_{2}$ are the coefficients describing the proportions from the total probed area. The reference spectrum of graphene is obtained from the measurement at the non-irradiated area of the sample. Then, the spectrum of GO can be extracted by using eq. 1 , if the coefficients $\mathrm{c}_{1}$ and $\mathrm{C}_{2}$ are known. First we consider the problem of the size of the probing beam. Since it is difficult to take into account the variation of the (Gaussian shaped) beam over the probed area explicitly, we divide the total beam energy into two regions corresponding mainly to the part which overlaps the oxidized area and the part which is outside the oxidized area. We estimate that the laser spot size is $500 \mathrm{~nm}$ (FWHM), while the spot size of the oxidized area is $300 \mathrm{~nm}$. Note that the oxidized spot size doesn't correspond exactly to the spot size estimated from Fig. 2(C). However, the oxidized spot size changes over irradiation time and the chosen value corresponds well with the asymptotic value of total oxidized area of Fig. 5(B). The proportion of the intensity of the probing beam outside the oxidized area was determined by integrating a 2dimensional Gaussian function between the limits $-\infty$ to $+\infty$ and between $-150 \mathrm{~nm}$ to $150 \mathrm{~nm}$, corresponding to the total intensity and the intensity of the beam inside the oxidized spot, respectively. The ratio of the intensity outside the oxidized area and inside the oxidized area was 
0.54. This means that for the fully oxidized spot the coefficient $c_{1}$ would be 0.54 and $c_{2}=0.46$. The second factor to consider is the fractional area of GO islands within the $300 \mathrm{~nm}$ spot. This information can be obtained from the fit of Fig. 5(B), which gives exactly this information. Accordingly, we extracted the intensities of the D- and G-bands and their ratio $I(D) / I(G)$ at different irradiation doses as follows:

$$
\begin{aligned}
& I^{G}(G)=1 \\
& I^{G}(D)=0.071 \\
& I^{G O}(G)=\frac{I^{O b s}(G)}{c_{2}}-\frac{c_{1}}{c_{2}} I^{G}(G) \\
& I^{G O}(D)=\frac{I^{O b s}(D)}{c_{2}}-\frac{c_{1}}{c_{2}} I^{G}(D)
\end{aligned}
$$

Where eq. (2) is arbitrary normalization and eq. (3) is determined by the reference spectrum of non-irradiated graphene. Equations (4) and (5) are obtained from eq. (1). Since all the spectra are measured in single mapping the measurement parameters are the same for all spectra. The peak intensities are determined as baseline-corrected heights, not areas, in order to match the analysis of Ref. 16. Since the quantity of interest is $\mathrm{I}(\mathrm{D}) / \mathrm{I}(\mathrm{G})$, further simplification can be made by taking the ratio of equations (5) and (4):

$$
\frac{I^{G O}(D)}{I^{G O}(G)}=\frac{I^{O b s}(D)-c_{1} I^{G}(D)}{I^{O b s}(G)-c_{1} I^{G}(G)}
$$

It should be noted that the analysis described in Eqs. (2) - (6) is strictly valid only for the presently used parameters and the values of the used coefficients should be evaluated separately 
for other situations. The result of the analysis is presented in Figure 9. The main observation is that the ratio rises constantly up to about $200(\mathrm{pJ})^{2}$ s after which the increase saturates.

Lucchese et al. quantified the behavior of $\mathrm{I}(\mathrm{D}) / \mathrm{I}(\mathrm{G})$ in graphene by controlled generation of defects with ion bombardment and analyzing the samples by STM and Raman spectroscopy. ${ }^{16}$ Up to the $\mathrm{I}(\mathrm{D}) / \mathrm{I}(\mathrm{G})$ ratio of $\sim 2$ corresponding to the defect density of $3 \times 10^{12} \mathrm{~cm}^{-2}$, the ratio can be described with the equation (7):

$\frac{I(D)}{I(G)}=\frac{102 \mathrm{~nm}^{2}}{L_{D}^{2}}$

where $L_{D}$ is the average distance between defects which is related to the density $\sigma$ by $L_{D}=\frac{1}{\sqrt{\sigma}}$. Although the numerical factor in Eq. (7) depends on the excitation wavelength ${ }^{17}$ it was derived using Raman excitation wavelength of $514 \mathrm{~nm}$, which is sufficiently close to $532 \mathrm{~nm}$ used in this work. We reproduce the obtained $L_{D}$ based on relation (7) in the inset of Fig. 9. The distance $L_{D}$ varies from $\sim 32 \mathrm{~nm}$ before the oxidation to $\sim 11 \mathrm{~nm}$ in the end, which corresponds to the defect density of $\sim 8 \times 10^{11} \mathrm{~cm}^{-2}$. It should be kept in mind that in our case "defect" is actually an oxidized (functionalized) site and the obtained defect density corresponds to the situation inside the oxidized islands. 


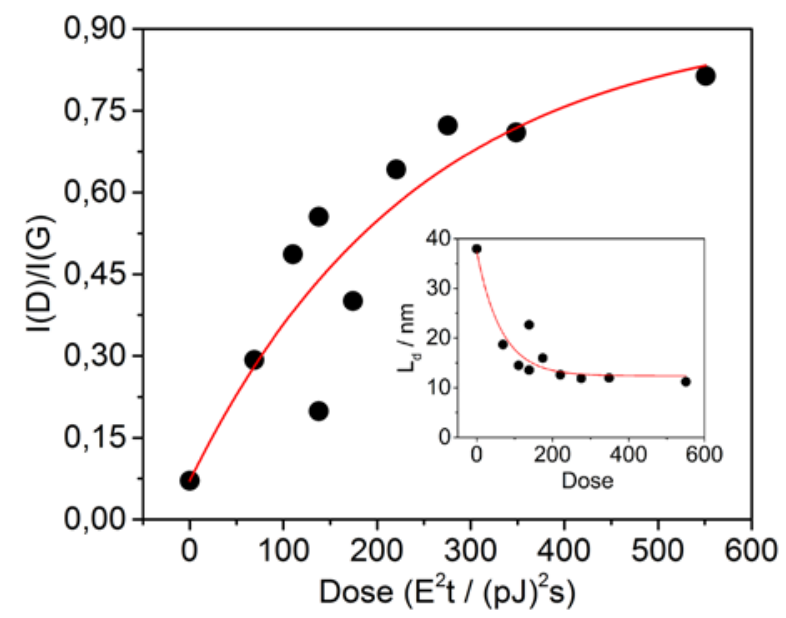

Figure 9. $\mathrm{I}(\mathrm{D}) / \mathrm{I}(\mathrm{G})$ ratio of the oxidized graphene as a function of irradiation dose. The fits are only for the guide to the eye.

\section{PHENOMENOLOGICAL MODEL}

Although the above results provide an overview of the growth kinematics at large length scales, their resolution is insufficient to provide understanding for the kinematics at the atomic scale. We therefore developed a microscopic growth model, a variant of the Eden model, ${ }^{18}$ which turned out to describe the experimental growth well. In the following we discuss the model in detail.

The structure and symmetry of the oxidized graphene is unknown, so we assume a square lattice with $h=2.5 \AA$ spacing. Given the two-photon process and the macroscopic total irradiation time $\tau$, the probability of oxidizing site $i$ is $\tau I(i)^{2} p_{i}$, where $I(i)$ is the laser intensity at site $i$ and $p_{i}$ is a relative oxidation probability that depends on the type of site. For pristine graphene sites away from islands $p_{i}=p_{p}$ and for edge sites next to islands $p_{i}=p_{e}$. As it will turn out, edge oxidation is much more probable than pristine oxidation, or $p_{e} \gg p_{p}$. To eliminate 
bias from lattice symmetry, the probability of oxidizing diagonal edge sites is reduced by a factor of $\sqrt{2}$. We choose units so that $I_{0}=1$ for maximum laser intensity and $p_{e}=1$ for the relative probability for oxidizing an edge site. This choice fixes the time scale so that in the middle of the laser spot an edge site oxidizes at the probability $\tau$ and a pristine site oxidizes at the probability $\tau p_{p}$, when the irradiation time is $\tau(\tau \ll 1)$.

We used this model to simulate laser irradiation by Gaussian laser spots with intensity $I(i)=I_{0} \exp \left[-\left(r_{i}-r_{0}\right)^{2} /\left(2 \sigma^{2}\right)\right]$, where $r_{0}$ is the spot center, $\sigma=F W H M /(2 \sqrt{2 \ln 2})$, and $F W H M=500 \mathrm{~nm}$ is the full width at half maximum. After testing the ratio $p_{p} / p_{e}$ for values spanning several orders of magnitude, the ratio $p_{p} / p_{e} \sim 10^{-5}$ turned out to give the best comparison both with the experimental oxidation patterns (compare Figs. 3 and 10) and with the island size distributions (compare Figs. 4 and 11). This result offers the first insight to the microscopic oxidation events: the oxidation of an edge site is five orders of magnitude more probable than the oxidation of a pristine site. 

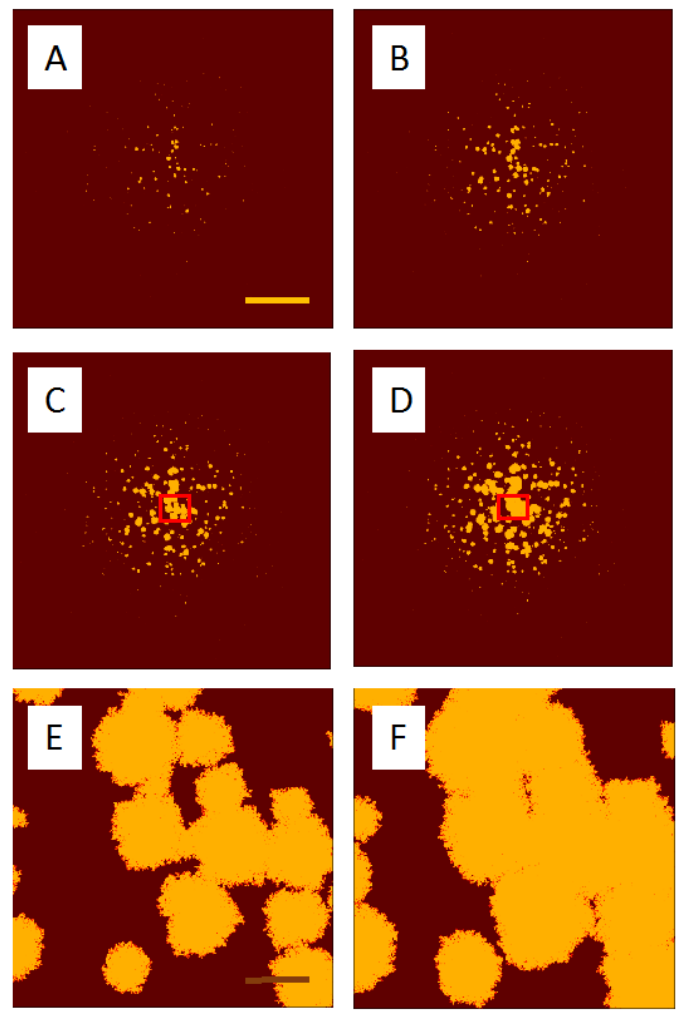

Figure 10. Simulated oxidation using a Gaussian laser profile (FWHM=500 nm) and irradiation time of (A) $\tau=13$, (B) $\tau=17$, (C) $\tau=22$, and (D) $\tau=26$. Panels $E$ and F show enlarged central parts of panels C and D, respectively. Scale bars $200 \mathrm{~nm}$ (A-D) and $20 \mathrm{~nm}$ $(\mathrm{E}, \mathrm{F})$.
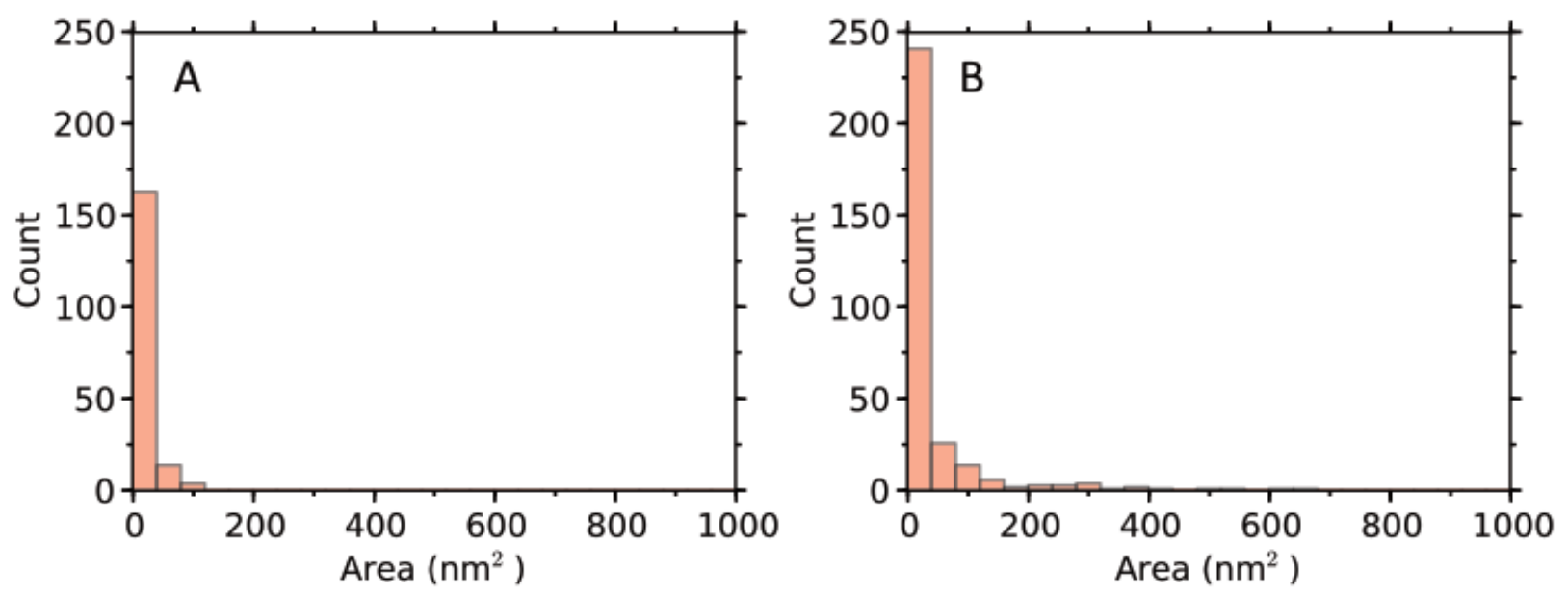
Figure 11. Island size distributions with a Gaussian laser profile (FWHM=500 nm) after irradiation time (A) $\tau=13$ and (B) $\tau=22$. (Panels A and B correspond to Figs. 10(A) and 10(C), respectively).

The model can provide further insight into the growth kinematics via analytical approach. Given the dominant edge growth, each created island $k$ grows at the rate $d n_{k} / d t=n_{e d g e, k} p_{e}$, where $n_{k}$ is the island size (number of oxidized sites) and $n_{e d g e, k}$ is the number of surrounding edge sites. The edge undergoes kinetic roughening, ${ }^{19}$ implying a scaling $n_{\text {edge, } k} \sim \lambda n_{k}^{\chi}$ between the edge length and the island size. A fit to the edge profiles of islands grown by simulation gave scaling parameters $\lambda=10$ and $\chi \approx 1 / 2$. For perfectly round islands with smooth edges these parameters would have been $\lambda=2 \sqrt{\pi} \approx 3.5$ and $\chi=1 / 2$, which implies that the microscopic edge length was nearly three times the apparent length viewed at the experimental resolution.

Let us then consider a pristine lattice with $N$ sites and ignore finite laser spot size by assuming a homogeneous intensity, $I(i)=I_{0}$. Islands are initially created at a rate $N p_{p}$ so that at time $t$ there are $N p_{p} t$ islands with sizes $n_{k}(t)=\left[\frac{1}{2} \lambda p_{e}\left(t-t_{k}\right)\right]^{2}$, where $t_{k}$ is the time island $k$ was created. Assuming a small density of islands for their independent growth, the total number of oxidized sites at time $t$ becomes

$$
n(t)=N p_{p}\left(\lambda p_{e}\right)^{2} t^{3} / 12
$$

as obtained by integrating over all islands $\left(n=\sum_{k} n_{k}\right)$. This growth regime signifies the slow induction period. 
The induction period ends and the growth kinematics change when the expanding islands begin to coalesce. This happens when approximately one-third of the sites are oxidized, $n\left(t^{\prime}\right) \approx$ $N / 3$. At this moment $t=t^{\prime}$ the growth of the existing $N^{\prime}=N p_{p} t^{\prime}$ islands begin to dominate the kinematics over the creation of new islands, which suggests that further oxidation can be modeled by the closing up approximately of $N^{\prime}$ holes (because $p_{e} / p_{p} \gg 1$ ). The total edge length in the holes is $n_{\text {edge }}=\beta \sqrt{N^{\prime}(N-n)}$, where the parameter $\beta$ is determined by requiring equal growth rate before and after $t^{\prime}$. Thus the total number of oxidized sites after the induction period $\left(t>t^{\prime}\right)$ becomes

$n(t)=N-\left(\sqrt{2 N / 3}-\frac{1}{2} p_{e} \beta \sqrt{N^{\prime}}\left(t-t^{\prime}\right)\right)^{2}$.

Equations (9) and (10) agree well with simulations (Fig. 12(A)) and capture the essential features in the growth kinematics despite the coarse analytical treatment. In particular, also the experimental statistics indicate traces of upward curvature (induction period) and subsequent saturation (Fig. 5(B)).

During the induction period, with overall oxidized area given by Eq. (9), the island area distribution is

$f\left(a_{k}\right)=\frac{p_{p}}{p_{e}} \frac{N}{\lambda h} \frac{1}{\sqrt{a_{k}}}$

where $a_{k}=h^{2} n_{k}$ and the upper limit for the distribution is determined by islands created in the very beginning (with $t_{k}=0$ ). It is straightforward to obtain a discrete version of Eq. (11), which 
agrees well with the simulations (Fig. 12(B)). Note that the distribution is in fair agreement even with the experimental distributions (Fig. 4), even though it ignores the finite laser spot size.
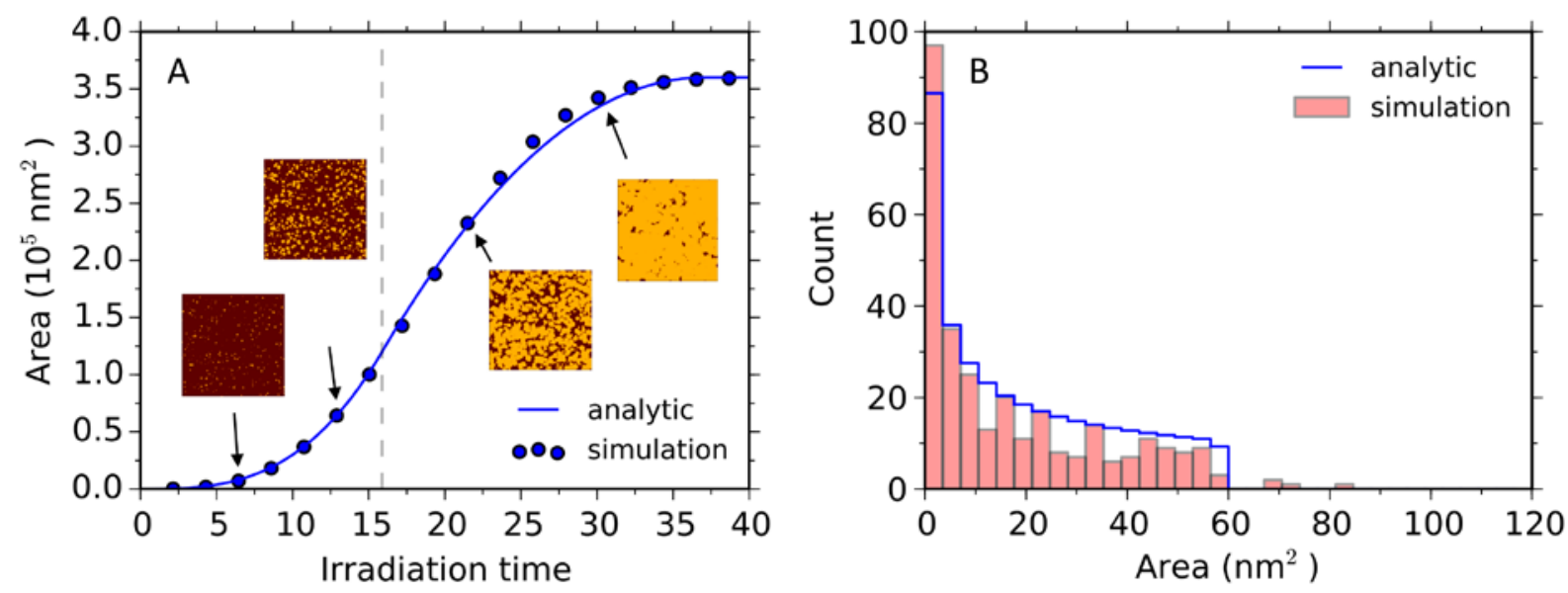

Figure 12. Simulated growth kinematics under homogeneous laser irradiation of $600 \mathrm{~nm} \times 600$ nm area. (A) Simulated values (spheres) and analytic estimates [line; Eqs. (9) and (10)] for the total oxidized area. Insets show examples of resulting oxidized patterns. Dashed vertical line denotes the end of induction period at $t^{\prime}$. (B) Island area distribution after irradiation time $\tau=7$ (corresponds to the first inset in panel a).

The model allows also further consistency checks with the experiment. The density of islands is approximately $\rho=N^{\prime} /\left(N h^{2}\right)$, as determined by the number of created islands and the end of the induction period. A straightforward calculation gives $\rho=6 \times 10^{14} \times\left(p_{p} / p_{e}\right)^{2 / 3} \mathrm{~cm}^{-2}$, or $\rho=3 \times 10^{11} \mathrm{~cm}^{2}$, which is in fair agreement with the experimentally estimated density of $\sim 10^{11}$ $\mathrm{cm}^{-2}$. Moreover, the correspondence of the experimental parameters is such that $I_{O}$ is proportional to pulse energy and $\tau$ is proportional to irradiation time. By comparing the model and the experiment (Figs. 5(B) and 12(A)), we can deduce an approximate correspondence $I_{0}^{2} \tau \widehat{=} 10 \times E^{2} t /\left(\left(\mathrm{pJ}^{2}\right) \mathrm{s}\right)$ between the model and experimental parameters, where $E$ is pulse 
energy and $t$ the experimental irradiation time. This correspondence could be used later to tune experimental parameters for custom-made properties of the oxidized islands. Note that while the pulsed nature of the laser is important for the oxidation mechanism, it is irrelevant for the modeling of growth kinematics.

The model can be used also to reproduce more complicated experimental exposure patterns. In order to test this we prepared a square array of oxidation spots by using a step size of $100 \mathrm{~nm}$ between the spots in a 21 x 21 matrix. The pulse energy was $13.2 \mathrm{pJ}$ and irradiation time $0.2 \mathrm{~s}$ per spot. The AFM image of the square is shown in Figure 13(A). It is interesting that the edges of the square are very sharp and lead to a well-defined geometrical structure. This shows that the oxidation method can be used to fabricate structures with a high degree of control. Figure 13(B) shows a magnified image of Fig. 13(A). A simulation of the structure is shown in Fig. 13(C) and the magnified view in Fig 13(D). The essential features such as island structure and sharp edges are well reproduced by the simulation. Thus, the model can be useful in designing oxidation patterns and their topographical properties. 
(A)

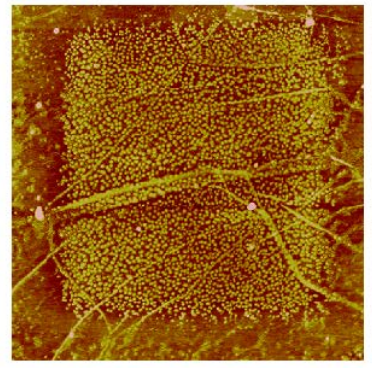

(C)

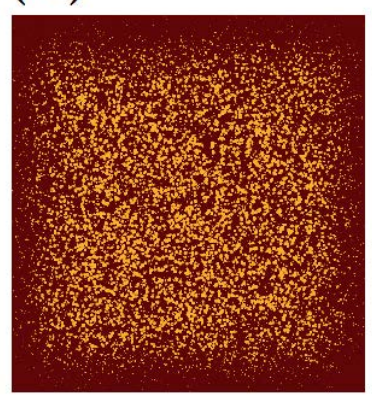

(B)

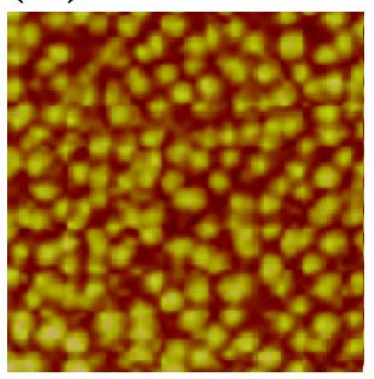

(D)

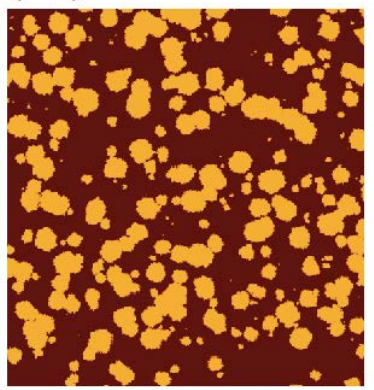

Figure 13. A) Square array made by oxidizing a matrix of spots with $100 \mathrm{~nm}$ spacing. B) A magnified view of a square array. C) Simulated square array. D) Magnified view of the simulated square array.

\section{DISCUSSION}

The results obtained from AFM, Raman and theoretical modelling yield a consistent picture of the mechanism of oxidation in the two-photon induced process. We start the discussion of the process from the lowest dose case. Raman data shows that already after the first irradiation the G-band shifts and narrows significantly and the 2D-band narrows. At this point the D-band has not grown much and the AFM images show mainly cleaning of residues and the appearance of the first seeds of oxidized islands. Thus, the behavior of the Raman bands at this stage reflects a process different from the actual oxidation which dominates at higher irradiation doses, and which is characterized by the growth of the D-band and up-shift of the G-band, characteristic for 
graphene oxide. ${ }^{13}$ Raman observations at low dose thus cannot be explained by oxidation but instead by electrostatic doping effects. ${ }^{14,15,20}$ The increase of $\omega_{0}(G)$ (G-band Raman shift) and the simultaneous decrease of $\mathrm{FWHM}(\mathrm{G})$ is a clear indication of a change in Fermi Energy originating from doping, although the type of doping is not deducible from the shift of the Gband. It should be noted that the position of the G-band for non-irradiated graphene $\left(1590.5 \mathrm{~cm}^{-}\right.$ ${ }^{1}$ ) indicates that already the starting material is doped. ${ }^{20}$ This is fairly common situation in the literature and it has been explained by physisorption of oxygen molecules which is assisted by water. ${ }^{21-25}$ In this situation, charge transfer between graphene and oxygen leads to p-type doping. Judging from the shift of the G-band our samples are doped at a level of $\sim 5 \times 10^{12} \mathrm{~cm}^{-2}{ }^{20}$ Within this assumption we can try to interpret the additional doping induced by irradiation and evidenced by further up-shift of the G-band. It corresponds either to additional p-type doping up to a total level of $\sim 8 \times 10^{12} \mathrm{~cm}^{-2}$ or to conversion of graphene to n-type doping up to a level of $\sim 1.6 \times 10^{13} \mathrm{~cm}^{-2}$. It has been suggested that irradiation with $532 \mathrm{~nm}$ light of graphene in the presence of water leads to p-type doping presumably due to water-assisted photoinduced chargetransfer leading to negative oxygen ions. ${ }^{22}$ This is the most plausible explanation in our case as well. The direction of the shift of the 2D-band can be used to test this hypothesis. General behavior leads to blue-shift for p-type and red-shift for n-type doping, but the shift is very small over a large range of both types of doping. ${ }^{20}$ In the range of doping estimated from the G-band the 2D-band should down-shift by about $1 \mathrm{~cm}^{-1}$, while an opposite behavior by a similar amount is observed. However, the observed shift is so small that taken into account other possible effects occurring it should not be taken as a strong argument against p-type doping. The decrease of the FWHM of both bands is in agreement with the p-type doping. The behavior at higher doses is not straightforward to interpret since oxidation leads to strong chemical modification of graphene 
and this affects the vibrational frequencies by different mechanisms than electrostatic doping. However, more important than finding out the exact doping mechanism is to recognize that all the evidence points to the presence of weakly bound oxygen and water on graphene. This observation is important since it provides an initial configuration for photo-oxidation of graphene, which is the central topic of this study and the focus of the following discussion.

As it is evident from the AFM images, graphene oxidation proceeds via formation of initial seeds, their growth to nanosized islands and further formation of new seeds. As the developed phenomenological model indicates, the probability of formation of new seeds is five orders of magnitude lower than the growth rate of the existing islands. This is a very important feature of the process as it leads to the characteristic morphology of the material. Although this mechanism is in qualitative agreement with previous observations that oxidation occurs preferentially at defect sites and grain boundaries, ${ }^{21,26}$ or previously oxidized sites, our model provides an estimate of the quantitative difference. This mechanism of oxidation is rather unique. UV-photooxidation leads to homogeneous decay of graphene ${ }^{27}$ and thermal oxidation leads to degradation of graphene and formation of holes and pits. ${ }^{21,26}$ Recently, a study was reported in which photothermal oxidation of graphene under controlled oxygen-containing atmosphere was shown to lead to smooth oxidation without formation of pores. ${ }^{28}$ In addition, oxidized areas were found to form nanosized patches, similarly to our case. However, the mechanisms in the two cases are different since our process is a "cold" process based on the extremely low average power of irradiation $(5-10 \mu \mathrm{W})$. In the ultrafast timescale, the temperatures get very high, up to several thousands of degrees of Kelvin, but the relaxation occurs also in the picosecond timescale. Photon absorption excites electrons from the valence to the conduction band. Electrons and holes thermalize within 100 fs and cool down simultaneously heating the phonon bath in the sub- 
picosecond timescale. Finally, cooling occurs in the picosecond timescale. ${ }^{29}$ Thus, most of the time, the sample remains close to room temperature. The main source of transient heating is linear absorption, which is expected to dominate photon absorption. The fact that two-photon behavior was observed for the oxidation mechanism ${ }^{9}$ shows that the process cannot be simple thermal reaction.

The analysis of the D-band indicates that oxidation does not proceed to the stage when oxidized material becomes highly disordered as it would be evidenced by strong broadening of the D-band and decrease of the I(D)/I(G) ratio after the initial increase. ${ }^{16}$ This, in turn, indicates that after reaching a certain level of oxidation inside the islands, the process proceeds in the graphene-graphene oxide interface but it is self-limited to a level of $\sim 8 \times 10^{11} \mathrm{~cm}^{-2}$ defect density inside the islands $\left(1 / \mathrm{L}_{\mathrm{D}}{ }^{2}\right.$ ), as determined from the $\mathrm{I}(\mathrm{D}) / \mathrm{I}(\mathrm{G})$ ratio (max value $\sim 0.8$, see Fig. 9. This is an important result since it indicates that the oxide material has uniform composition within the islands. In fact, the density corresponds to a fairly small number of defects inside an island with a typical size of $30 \mathrm{~nm}$, namely 6. It means that the initial seeds actually are probably just point functionalizations. The self-limitation can be explained by changing optical properties leading to reduced probability for photo-oxidation. Some hint to this is provided by FWM imaging which shows via image contrast provided by oxidation that nonlinear response is very sensitive to the level of oxidation. In particular, oxidized graphene shows reduced FWM response. Upon prolonged irradiation, finally, material degrades, as is evident by the morphology of heavily irradiated spots which show flattened central part.

The size of islands before coalescence is about $30 \mathrm{~nm}$, corresponding to a density of $1.1 \times 10^{11}$ $\mathrm{cm}^{-2}$. It is interesting to note that the density of oxide islands is similar to the density of "charge puddles” observed to exist in graphene supported on $\mathrm{SiO}_{2}{ }^{30,31}$ There have been reports on the 
correlation between the presence of charge puddles and thermal oxidation of graphene. ${ }^{26}$ Our observations provide additional evidence of this correlation and suggests a link between charge puddles, adsorbed oxygen and two-photon oxidation. However, more studies are needed to put this link on a more quantitative basis.

There have been several previous reports on laser induced oxidation of graphene. Herziger et al. studied CW laser irradiation induced oxidation by Raman spectroscopy. ${ }^{32}$ Their mechanism is based on laser induced heating of the sample and subsequent thermal oxidation of graphene whereas in our case the extremely low average power of $5-10 \mu \mathrm{W}$ cannot lead to significant heating of the sample. A study by Islam et al. involved also a CW laser induced photothermal mechanism of oxidation. ${ }^{28}$ There is yet another report of CW laser induced oxidation of graphene by a green $(532 \mathrm{~nm})$ laser. $^{22}$ In this case the authors estimate that the sample heating is only about 100 degrees which is too low for thermal oxidation. They observed very slow oxidation in timescale of hours as evidenced by the slow growth of the D-band. Water was found to be essential for oxidation. Rapid G-band upshift in a much faster timescale than the growth of the D-band was observed, similarly to our case. It is interesting to estimate if the two-photon mechanism could explain also oxidation under CW irradiation. In Ref. 22 laser power was 7.7 $\mathrm{mW}$ and the spot diameter was $5 \mu \mathrm{m}$ yielding intensity $\mathrm{I}=3.9 \times 10^{4} \mathrm{Wcm}^{-2}$. In our case the intensity is $1.3 \times 10^{11} \mathrm{Wcm}^{-2}$ (for $10 \mathrm{pJ}$ pulse energy) but the illumination time of the sample is only $2.4 \times 10^{-8}$ s per second due to the short pulses (40 fs) and $600 \mathrm{kHz}$ repetition rate. Assuming that photo-oxidation is due to two-photon process, the rate of oxidation is proportional to $\sigma^{(2)} \mathrm{I}^{2}$, where $\sigma^{(2)}$ is a two-photon cross-section and I is intensity. To compare the observed rates we calculate $\sigma^{(2)} \mathrm{I}^{2} \Delta \mathrm{t}$, where $\Delta \mathrm{t}$ is illumination time per $1 \mathrm{~s}$. Taking the ratio of this quantity between CW and pulsed illumination yields that CW photo-oxidation in Ref. 22 should be $\sim 3 \times 10^{5}$ times 
slower than in our case. In ref 22 the rate of change of the $\mathrm{I}(\mathrm{D}) / \mathrm{I}(\mathrm{G})$ ratio as a function of irradiation time was reported and when comparing corresponding values for our case yields that the change is slower by a factor of $1.2 \times 10^{6}$ or $9 \times 10^{3}$ for dry and wet graphene, respectively. The value predicted by assuming a common two-photon mechanism is between these limits and thus slow CW photo-oxidation in Ref. 22 indeed may be due to two-photon mechanism.

Understanding the mechanism of oxidation leads to the possibility for tuning the material properties. As shown in Fig. 13 the experiment and simulation yield similar structures for extended irradiated area. Judging from Figs. 13(B,D), the structure of the material can be described as an irregular nanomesh. ${ }^{33}$ In this case the size distribution of the islands is fairly narrow and it shows how properties of the material can be tuned by choosing suitable irradiation parameters. This kind of a nanomesh is interesting since it provides two levels of control over the electrical properties. The narrow graphene channels in between the oxide islands resemble nanoribbons and their width determines the opening of a band gap which gets larger at smaller dimensions. ${ }^{32}$ The second level of control is provided by the stage of coalescence of the islands and closing of the graphene channels. At this point the electrical properties are determined by graphene oxide which behaves like a semiconductor or insulator depending on the level of oxidation. ${ }^{11,12}$ This control of electrical properties is in agreement with our previous report where we observed gradual increase of resistance and finally opening of a band gap and finally formation of insulator at increasing irradiation dose. ${ }^{9}$ The present results give valuable insight into the oxidation mechanism and morphology of the oxidized graphene opening a new avenue for controlled tailoring of properties of graphene for electronic and photonic applications.

Two-photon oxidation offers benefits compared to chemical oxidation. Patterning in submicron scale can be done simply with direct laser writing. Additionally, the oxidized material is 
more ordered, as evidenced by Raman spectroscopy. Finally, in patterning, two-photon oxidation is superior to laser reduction of graphene oxide, as reduction does not recover the excellent properties of graphene due to remaining oxidized groups.

\section{CONCLUSIONS}

Two-photon induced oxidation of graphene was studied by systematic oxidation and AFM and Raman investigations. The key observations are: i) oxidation starts from small seeds which grow to islands which finally coalesce together; ii) the functionalization density inside the oxide islands first grows and then remains constant at the level of $8 \times 10^{11} \mathrm{~cm}^{-2}$ and the oxidation progresses in the graphene-graphene oxide interface; iii) according to the developed phenomenological model the probability for oxidation next to the already oxidized site is five orders of magnitude higher than oxidation in pristine graphene; iv) Extended areas with relatively narrow size distribution of islands and sharp area edges can be made by using suitable irradiation parameters yielding irregular nanomesh of oxide islands surrounded by graphene nanoribbons.

The developed understanding of the mechanism of oxidation gives important information that can be used to tune electrical properties of oxidized graphene, which can be further exploited in development of graphene devices.

\section{ACKNOWLEDGMENT}

L.S. thanks the financial support by the Ministry of Education, Youth and Sports of the Czech Republic under the project CEITEC 2020 (LQ1601) and the support of the grant STI-S-14-523 
(Advanced Nanotechnologies and Materials). Sutidara Nopakun is thanked for measuring the data for Fig. 13(A,B). P.K. acknowledges the Academy of Finland for funding (Projects No. $283103 \& 251216)$.

\section{AUTHOR INFORMATION}

\section{Corresponding Author}

*Mika Pettersson, Email: mika.j.pettersson@jyu.fi, Tel: +358 503109969

\section{Author Contributions}

The manuscript was written through contributions of all authors. All authors have given approval to the final version of the manuscript.

\section{REFERENCES}

(1) Weiss, N. O.; Zhou, H.; Liao, L.; Liu, L.; Jiang, S.; Huang, Y.; Duan, X. Graphene: An Emerging Electronic Material. Adv. Mater. 2012, 24, 5782 - 5825.

(2) Bao, Q.; Loh, K. P. Graphene Photonics, Plasmonics, and Broadband Optoelectronic Devices. ACS Nano, 2012, 6, 3677 - 3694.

(3) Pasanen, P.; Voutilainen, M.; Helle, M.; Song, X.; Hakonen, P. Graphene for Future Electronics. Phys. Scr. 2012, T146, 014025. 
(4) Zhang, Y.; Guo, L.; He, Y.; Xia, H.; Chen, Q.; Sun, H.-B.; Xiao, F.-S. Direct Imprinting of Microcircuits on Graphene Oxides Film by Femtosecond Laser Reduction. Nano Today, 2010, 5, $15-20$.

(5) Guo, L.; Shao, R.-Q.; Zhang, Y.-L.; Jiang, H.-B.; Li, X.-B.; Xie, S.-Y.; Xu, B.-B.; Chen, Q.-D.; Song, J.-F.; Sun, H.-B. Bandgap Tailoring and Synchronous Microdevices Patterning of Graphene Oxides. J. Phys. Chem. C, 2012, 116, 3594 - 3599.

(6) Strong, V.; Dubin, S.; El-Kady, M. F.; Lech, A.; Wang, Y.; Weiller, B. H.; Kaner, R. B. Patterning and Electronic Tuning of Laser Scribed Graphene for Flexible All-Carbon Devices. ACS Nano, 2012, 6, 1395 - 1403.

(7) El-Kady, M. F.; Strong, V.; Dubin, S.; Kaner, R. B. Laser Scribing of High-Performance and Flexible Graphene-Based Electrochemical Capacitors. Science, 2012, 335, 1326 - 1330.

(8) El-Kady, M. F.; Kaner, R. B. Direct Laser Writing of Graphene Electronics. ACS Nano, 2014, 8, $8725-8729$.

(9) Aumanen, J.; Johansson, A.; Koivistoinen, J.; Myllyperkiö, P.; Pettersson, M. Patterning and Tuning of Electrical and Optical Properties of Graphene by Laser Induced Two-Photon Oxidation. Nanoscale, 2015, 7, 2851 - 2855.

(10) Koivistoinen, J.; Aumanen, J.; Hiltunen, V.-M.; Myllyperkiö, P.; Johansson, A.; Pettersson, M. Real-Time Monitoring of Graphene Patterning with Wide-Field Four-Wave Mixing Microscopy. Appl. Phys. Lett. 2016, 108, 153112. 
(11) Jung, I.; Dikin, D. A.; Piner, R. P.; Ruoff, R. S. Tunable Electrical Conductivity of Individual Graphene Oxide Sheets Reduced at "Low” Temperatures. Nano Lett. 2008, 8, 4283 4287.

(12) Yan, J.-A.; Xian, L.; Chou, M. Y. Structural and Electronic Properties of Oxidized Graphene. Phys. Rev. Lett. 2009, 103, 086802.

(13) Perrozzi, F.; Prezioso, S.; Ottaviano, L. Graphene Oxide: From Fundamentals to Applications. J. Phys.: Condens. Matter, 2015, 27, 013002.

(14) Ferrari, A. C. Raman Spectroscopy of Graphene and Graphite: Disorder, Electron-Phonon Coupling, Doping and Nonadiabatic Effects. Sol. State Comm. 2007, 143, 47 - 57.

(15) Beams, R.; Cançado, L. G.; Novotny, L. Raman Characterization of Defects and Dopants in Graphene. J. Phys. Condens. Matter 2015, 27, 083002.

(16) Lucchese, M. M.; Stavale, F.; Martins Ferreira, E. H.; Vilani, C.; Moutinho, M. V. O.; Capaz, R. B.; Achete, C. A.; Jorio, A. Quantifying Ion-Induced Defects and Raman Relaxation Length in Graphene. Carbon, 2010, 48, 1592 - 1597.

(17) Matthews, M. J.; Pimenta, M. A.; Dresselhaus, G.; Dresselhaus, M. S.; Endo, M. Origin of Dispersive Effects of the Raman D Band in Carbon Materials. Phys. Rev. B, 1999, 59, R6585.

(18). M. Eden, Proceedings of the $4^{\text {th }}$ Berkeley symposium on Mathematical Statistics and Probability 1961, 4, 223.

(19). Miyazima, S.; Yoshinaga, H.; Nagamine, T.; Takano, H.; Kertész, J.; Stanley, H. E. Growth Properties of Eden Model With Acceleration Sites. Physica A, 1998, 259, 1-9. 
(20) Das, A.; Pisana, S.; Chakraborty, B.; Piscanec, S.; Saha, S. K.; Waghmare, U. V.; Novoselov, K. S.; Krishnamurthy, H. R.; Geim, A. K.; Ferrari, A. C.; Sood, A. K. Monitoring Dopants by Raman Scattering in an Electrochemically Top-Gated Graphene Transistor. Nat. Nanotech. 2008, 3, $210-215$.

(21) Liu, L.; Ryu, S.; Tomasik, M. R.; Stolyarova, E.; Jung, N.; Hybertsen, M. S.; Steigerwald, M. L.; Brus, L. E.; Flynn, G. W. Graphene Oxidation: Thickness-Dependent Etching and Strong Chemical Doping. Nano Lett. 2008, 8, 1965 - 1970.

(22) Mitoma, N.; Nouchi, R.; Tanigaki, K. Photo-Oxidation of Graphene in the Presence of Water. J. Phys. Chem. C, 2013, 117, 1453 - 1456.

(23) Ryu, S.; Berciaud, S.; Yu, Y.-J.; Kim, P.; Flynn, G. W.; Brus, L. E. Atmospheric Oxygen Binding and hole Doping in Deformed Graphene on a $\mathrm{SiO}_{2}$ Substrate. Nano Lett. 2010, 10, 4944 $-4951$.

(24) Yang, Y.; Murali, R. Binding Mechanism of Molecular Oxygen and Moisture to Graphene. Appl. Phys. Lett. 2011, 98, 093116.

(25) Berholts, A.; Kahro, T.; Floren, A.; Alles, H.; Jaaniso, R. Photo-Activated Oxygen Sensitivity of Graphene at Room Temperature. Appl. Phys. Lett. 2014, 105, 163111.

(26) Yamamoto, M.; Einstein, T. L.; Fuhrer, M. S.; Gullen, W. G. Charge Inhomogeneity Determines Oxidative Reactivity of Graphene on Substrates. ACS Nano 2012, 6, 8335 - 8341.

(27) Zhao, S.; Surwade, S. P.; Li, Z.; Liu, H. Photochemical Oxidation of CVD-Grown Single Layer Graphene. Nanotech. 2012, 23, 355703. 
(28) Islam, A. E.; Kim, S. S.; Rao, R.; Ngo, Y.; Jiang, J.; Nikolaev, P.; Naik, R.; Pachter, R.; Boeckl, J.; Maruyama, B. Photo-Thermal Oxidation of Single Layer Graphene. RSC Adv. 2016, $6,42545-42553$.

(29) Malic, E. and Knorr, A. Graphene and Carbon Nanotubes, Ultrafast Optics and Relaxation Dynamics; Wiley-VCH, Weinheim, Germany, 2013.

(30) Martin, J.; Akerman, N.; Ulbricht, G.; Lohmann, T.; Smet, J. H.; von Klitzing, K.; Yacoby, A. Observation of Electron-Hole Puddles in Graphene Using a Scanning SingleElectron Transistor. Nat. Phys. 2008, 4, 144 - 148.

(31) Zhang, Y.; Brar, V. W.; Girit, C.; Zettl, A.; Crommie, M. F. Origin of Spatial Charge Inhomogeneity in Graphene. Nat. Phys. 2009, 5, 722 - 726.

(32) Herziger, F.; Mirzayev, R.; Poliani, E.; Maultzsch, J. In-Situ Raman Study of LaserInduced Graphene Oxidation. Phys. Stat. Sol. B, 2015, 1-5.

(33) Bai, J.; Zhong, X.; Jiang, S.; Huang, Y.; Duan, X. Graphene Nanomesh. Nat. Nanotech. 2010, 5, 190 - 194.

Table of Contents Graphic 


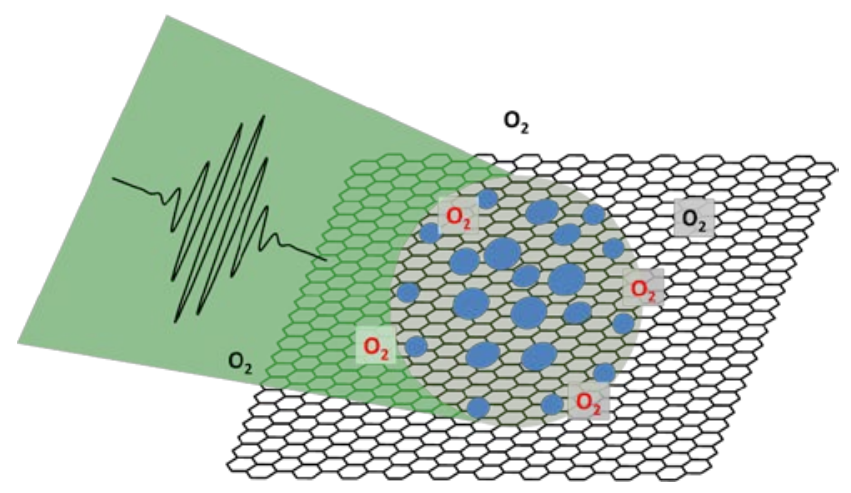

